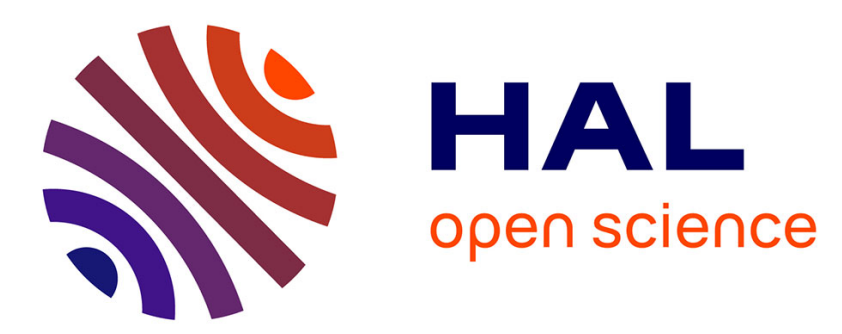

\title{
L'Accord de Paris : fin de la crise du multilatéralisme climatique ou évolution en clair-obscur?
}

Sophie Lavallée, Sandrine Maljean-Dubois

\section{To cite this version:}

Sophie Lavallée, Sandrine Maljean-Dubois. L'Accord de Paris: fin de la crise du multilatéralisme climatique ou évolution en clair-obscur?. Revue Juridique de l'Environnement, 2016, 1, pp. 19-36. hal-01400330

\section{HAL Id: hal-01400330 \\ https://hal.science/hal-01400330}

Submitted on 21 Nov 2016

HAL is a multi-disciplinary open access archive for the deposit and dissemination of scientific research documents, whether they are published or not. The documents may come from teaching and research institutions in France or abroad, or from public or private research centers.
L'archive ouverte pluridisciplinaire HAL, est destinée au dépôt et à la diffusion de documents scientifiques de niveau recherche, publiés ou non, émanant des établissements d'enseignement et de recherche français ou étrangers, des laboratoires publics ou privés. 


\title{
L'Accord de Paris : fin de la crise du multilatéralisme climatique ou évolution en clair- obscur?
}

\author{
Sophie Lavallée ${ }^{(*)}$
}

\section{Sandrine Maljean-Dubois ${ }^{(*)}$}

En tant que problème politique et moral, le réchauffement de la planète est un défi global, c'est-à-dire une menace physique qui peut frapper gravement ou mortellement tout le monde sur terre et que l'on ne contrera avec quelque chance de succès que si la quasi - totalité des pays et des peuples de la planète agissent ensemble. Seules les armes nucléaires approchent de tels critères de définition, même si l'on tient compte des différences entre ces deux menaces qui, ensemble, représentent la fin de la rationalité politique moderne et la transition vers une ère nouvelle et inconnue $\quad(. .$.$) . ( F$. Cerutti, «Le réchauffement de la planète et les générations futures», Pouvoirs, $\mathrm{n}^{\circ} 127$ - Droit et environnement - novembre 2008 - p. 107-122, p. 108-109).

Alors que plusieurs se félicitent qu'un texte engageant pour la première fois tous les pays onusiens à lutter contre le réchauffement climatique ait pu être adopté dans la Ville Lumière, d'autres dénoncent l'inefficacité du processus qui a conduit à l'adoption d'un accord faible qui ne modifiera pas l'actuelle trajectoire d'un réchauffement climatique dangereux de $3^{\circ}$ ou $3,5^{\circ}$ Celsius. Entre ombres et lumières, le processus de négociation ayant mené à la conclusion de l'Accord de Paris a-t-il mis fin à la crise du multilatéralisme climatique ? Est-il le point de départ d'une nouvelle ère ? Va-t-il nous placer sur la bonne trajectoire d'émissions ? Afin d'analyser cette question, il convient de souligner qu'après la Conférence de Copenhague (COP15) de 2009, la Convention-cadre des Nations Unies sur les changements climatiques ${ }^{1}$ (CCNUCC) est redevenue le centre de gravité des négociations ayant mené à l'Accord de Paris (I), ce qui ne peut se comprendre qu'à la faveur de considérations politiques et économiques qui ont imprimé leur marque sur la forme et le contenu du texte (II).

${ }^{(*)}$ Professeure de droit, Faculté de droit, Université Laval, Québec, Canada, Chercheure au Centre de droit international et transnational de l'Université Laval (CDIT) et au Centre de la science de la biodiversité du Québec (CSBQ). Membre de l'Institut Environnement, Développement, Société (EDS) et de l'Institut des hautes études internationales (HEI), Québec.

${ }^{(* *)}$ Directrice de recherche au CNRS, Aix Marseille Univ, Université de Toulon, Univ Pau \& Pays Adour, CNRS, DICE, Aix-en-Provence, France. Ce travail a bénéficié du soutien de l'Agence nationale pour la recherche française dans le cadre du projet $<$ ANR-12-GLOB-0001-03 CIRCULEX $>$.

19 mai 1992, 1771 R.T.N.U. 107. 


\section{La CCNUCC : centre de gravité des négociations d'un futur accord}

Négocié pour être prolongé dans le temps, le Protocole de $K_{y o t o}{ }^{2}$ ne formulait pas d'obligations chiffrées de réduction des émissions de gaz à effet de serre pour les pays de son annexe $\mathrm{B}$, audelà d'une première période d'engagement des parties, qui a pris fin au 31 décembre 2012. II prévoyait que la Conférence des Parties siégeant en tant que Réunion des Parties au Protocole (COP/MOP) entame l'examen des engagements «sept ans au moins» avant la fin de la première période d'engagements allant de 2008 à 2012 (art. 3-9). Contrairement à ce qu'on envisageait au moment de la négociation du Protocole, ce n'est pas la COP/MOP du Protocole qui a été au cœur des négociations d'un accord Post-2012, mais bien la COP de la CCNUCC qui en a été le centre de gravité (1.1). Cela n'est pas étonnant quand on considère que la COP est un forum plus large, qui inclut les États-Unis et le Canada, deux grands émetteurs incontournables, et qu'elle pose les fondements de tout le régime climatique en en formulant les principes fondateurs, dont celui des responsabilités communes, mais différenciées. Elle permet aussi de dépasser la division binaire du Protocole de Kyoto et d'envisager que les grands pays émergents prennent également des engagements de réduction de leurs émissions. Dès lors, de la Conférence de Bali (COP13), en 2007, à celle de Paris de 2015 (COP21) en passant par les conférences de Poznan (COP14), Copenhague (COP15), Cancún (COP16), Durban (COP17), Varsovie (COP18), Lima (COP19) et Doha (COP20), les négociations pour la période postKyoto ont suivi deux processus parallèles. Le premier, appelé «Ad Hoc Working Group on Further Commitments for Annex I Parties under the Kyoto Protocol» («processus AWG-KP») a été mis en place en 2005, à la Conférence de Montréal, conformément à l'article 3.9 du Protocole de Kyoto (1.1). Le second a été établi en 2008, lorsque les parties à la CCNUCC ont adopté le Plan d'action de Bali $^{3}$, qui lançait un processus de négociation parallèle, appelé « $A d$ Hoc Working Group on Long-term Cooperative Action under the Convention («AWG-LCA») (1.2).

\subsection{Les négociations sous l'égide du Protocole de Kyoto}

Dès sa première réunion, la Conférence des Parties siégeant en tant que Réunion des Parties au Protocole de Kyoto a créé, en 2005, à Montréal, un Groupe de travail spécial des nouveaux

\footnotetext{
2 Protocole de Kyoto à la Convention-cadre des Nations Unies sur les changements climatiques, 11 décembre 1997, 2302 R.T.N.U. 148.

${ }^{3}$ CCNUCC 2008, Rapport de la treizième session de la Conférence des Parties tenue à Bali du 3 au 15 décembre, 14 mars 2008. 2007, FCCC/CP/2007/6/Add.1, 14 mars 2008.
} 
engagements des Parties visées à l'Annexe I au titre du Protocole de Kyoto. ${ }^{4}$ Ce Groupe à composition non limitée réunissait exclusivement les Parties au Protocole de Kyoto. Ce processus, ayant pour objectif d'aboutir à la négociation de nouveaux engagements des Parties visées à l'annexe I de la CCNUCC pour la période postérieure à 2012, excluait donc formellement les États-Unis, qui n'en étaient qu'observateurs. Ce groupe spécial devait faire rapport sur ses travaux à chaque COP/MOP et les achever «dans les meilleurs délais et suffisamment tôt pour éviter tout hiatus entre la première et la deuxième période d'engagement». ${ }^{5}$

Ainsi, dès la COP/MOP de Montréal, en 2005, les Parties au Protocole décidaient de négocier les modalités d'engagement des pays industrialisés pour les périodes suivantes, et ils se mettaient d'accord pour adopter l'amendement au Protocole avant la fin de l'année 2009 pour permettre aux États de le ratifier et d'ainsi éviter qu'il n'y ait, à l'échéance de la première période d'engagement, un «vide juridique et une démobilisation politique dans la lutte contre le changement climatique». ${ }^{6}$

L'absence des États-Unis a eu tôt fait de poser un problème insurmontable pour les négociations d'une deuxième période d'engagement du Protocole. Toujours résolus à ne souscrire à aucun engagement international de réduction des GES tant que les autres grands émetteurs comme la Chine, I'Inde, le Brésil, la Corée du Sud, l'Indonésie, l'Afrique du Sud et le Mexique ne s'engageraient pas à des obligations de réduction similaires, les États-Unis ont argué qu'une période d'engagement 2013-2020 ne servirait pas l'intérêt de l'humanité. Les premiers temps de la négociation ont mis en évidence que ces pays, tout en étant attachés au maintien du Protocole de Kyoto, ne souscriraient pas d'engagements dans ce cadre. Bien que les pays développés n'aient pas tous joué un rôle de chef de file dans la lutte contre les changements climatiques, alors même que la CCNUCC leur reconnaissait ce rôle (art. 3-1), on pouvait se demander à quoi serviraient les seuls efforts de réduction des pays industrialisés si on assistait à la poursuite de l'augmentation exponentielle des émissions de GES des pays émergents pendant une seconde période d'engagement du Protocole. Le groupe AWG-KP était

\footnotetext{
${ }^{4}$ Décision 1/CMP.1, étude au titre du paragraphe 9 de l'article 3 du Protocole de Kyoto des engagements des Parties visées à l'annexe $I$ de la Convention pour les périodes suivantes, FCCC/KP/CMP/2005/8/Add.130 mars 2006.

${ }^{5}$ Id., para. 3.

${ }^{6}$ Sandrine Maljean-Dubois et Matthieu Wemaëre, La diplomatie climatique de Rio 1992 à Paris 2015, Paris, Éditions Pedone, 2015, p. 163.
} 
dès lors dans une impasse. C'est ce qui a conduit au lancement de négociations dans le cadre plus inclusif de la CCNUCC.

La voie de négociation sous l'égide du Protocole, chère aux pays en développement parce qu'elle présentait des garanties quant au respect de la dualité des obligations de réduction des émissions de GES formalisée dans les annexes du Protocole, n'a ainsi pas conduit à un Protocole révisé à la Conférence de Copenhague, en 2009. II a fallu attendre la conférence de Durban, en 2011, pour que la continuation du Protocole après 2012 soit acceptée par les Parties au Protocole, en échange du lancement de nouvelles négociations sur le post-2020 dans le cadre de la CCNUCC. Cette feuille de route, devenue la «Plateforme de Durban pour une action concertée», a été proposée par l'Union européenne, composée alors de 27 États, et les pays les moins avancés et ceci, bien que le Japon, la Russie, le Canada et la Nouvelle-Zélande aient annoncé qu'ils ne s'engageraient pas dans une seconde période d'engagement du Protocole de Kyoto. Cette continuation ne réunira que l'Union européenne, I'Islande, la Croatie, Monaco, la Norvège, la Suisse et l'Australie, qui tous ensemble ne représentent guère qu'une part faible $13 \%$ - et déclinante des émissions mondiales de CO2. Son caractère restreint nécessite de chercher ailleurs - sur un plan politique - les raisons d'être de cette continuation toute symbolique.

En effet, en soutenant la conclusion d'une deuxième période d'engagement du Protocole, l'Union européenne jouait de stratégie, étant la seule à pouvoir la proposer face à l'absence des États-Unis comme partie au Protocole. Cette stratégie visait d'abord à éviter le «hiatus» entre la fin de la première période d'engagement et la seconde, en permettant au Protocole de s'appliquer de 2012 à 2020. Comme l'amendement de Doha n'est pas entré en vigueur, les parties l'ayant ratifié ont été invitées à l'appliquer transitoirement jusqu'en 2020. La stratégie européenne visait ensuite - et paradoxalement - à préserver les acquis du Protocole de Kyoto, notamment sur le plan de la différenciation, condition requise par les pays en développement.

C'est pourquoi à la conférence de Doha (COP), en 2012, la continuation du Protocole après 2012 ne pouvait pas être abandonnée, car cela aurait menacé l'ensemble des négociations post-2012. L'amendement de Doha au Protocole de Kyoto, dont l'entrée en vigueur nécessite la ratification de la majorité des trois quarts des Parties au Protocole ${ }^{7}$, est ainsi appelé à juste titre

\footnotetext{
${ }^{7}$ Protocole de Kyoto, art. 20. Le 12 janvier 2016, 59 parties l'ont ratifié, sur les 144 parties dont le vote est nécessaire à son entrée en vigueur.
} 
«passerelle climat de Doha» car il était conçu pour servir non seulement de pont entre la fin de la première période d'engagement et l'entrée en vigueur d'un futur accord qui serait adopté à Paris, mais également entre la voie de négociation sous l'égide du Protocole de Kyoto et la seconde sous l'égide de la Convention. Ainsi, la décision politique de continuer le Protocole audelà de 2012 était-elle importante pour soutenir les négociations pour l'après 2020 ; son adoption et celle de l'accord post-2020 peuvent ainsi être considérées comme un seul «paquet».

L'amendement de Doha visant la prolongation d'un Protocole marginalisé ne représentant que $13 \%$ des émissions mondiales, il était prévisible que le centre de gravité des négociations se déplace vers la conclusion d'un nouvel accord, plus ambitieux, car engageant tous les grands émetteurs, et qui serait négocié dans le cadre et sous l'égide de la CCNUCC.

\subsection{Les négociations sous l'égide de la Convention-cadre des Nations Unies sur les changements climatiques}

En décembre 1997, à la Conférence de Bali, la COP-13 crée un Groupe de travail spécial sur l'action concertée à long terme au titre de la Convention. Elle le charge de piloter le vaste processus qu'elle vient de lancer et lui fixe un calendrier de travail ainsi qu'une date pour la fin de ses travaux, soit la $15^{\mathrm{e}}$ Conférence des Parties, en 2009. Ce groupe de travail a pour mandat de mettre en œuvre le Plan d'action adopté à Bali, feuille de route vers Copenhague qui prévoit que les parties à la CCNUCC doivent réfléchir aux voies et moyens de renforcer la Convention sur des questions aussi essentielles que la vision commune de l'action collective, notamment l'objectif global à long terme de réduction des émissions, les engagements en matière d'atténuation, la coopération internationale pour appuyer la mise en œuvre de mesures d'adaptation, le transfert de technologies pour appuyer les mesures d'atténuation et d'adaptation et les ressources financières nouvelles et additionnelles en faveur des pays en développement. ${ }^{8}$

Le plan d'action de Bali soulevait des questions importantes : les négociations devraient-elles mener à deux accords ou «résultats», l'un en vertu du Protocole de Kyoto et l'autre en vertu de la CCNUCC, ou plutôt à un seul résultat qui rassemblerait les deux processus? Et cet ou ces accord(s) ou «résultat(s)» serai(en)t-il(s) de nature juridiquement contraignante ? Les pays en

\footnotetext{
${ }^{8}$ Plan d'action de Bali, FCCC/CP/2007/6/Add.1, Decision 1/CP.13, 14 mars 2008.
} 
développement ont fait bloc pour s'opposer à ce qu'un seul accord soit adopté en vertu de la CCNUCC plutôt que du Protocole de Kyoto. La négociation d'une seconde période d'engagement des pays développés de l'annexe B du Protocole de Kyoto était pour eux la seule solution juste et équitable. Les pays de l'Annexe I et les États-Unis ne considéraient, quant à eux, que l'autre alternative, soit la négociation d'un seul accord, juridiquement contraignant, qui fixerait les engagements des pays développés et des pays en développement. Cette position est d'ailleurs présente dans le Plan d'action de Bali, lequel visait à «permettre l'application intégrale, effective et continue de la Convention par une action concertée à long terme, dès à présent, d'ici à 2012 et au-delà (...)». ${ }^{9}$ Cette «feuille de route» vers Copenhague rappelait le principe des responsabilités communes, mais différenciées et mentionnait que l'action collective pour l'atténuation des changements climatiques devait envisager des engagements d'atténuation pour les «pays développés» et des «actions» pour les «pays en développement». ${ }^{10}$ Le Plan d'Action de Bali ne faisait donc plus référence aux annexes I et II de la Convention, ce qui pouvait laisser présager une autre différenciation pour la seconde période d'engagement que celle du Protocole de Kyoto. De plus, il ne prévoyait pas la forme juridique du «résultat» à atteindre dans le cadre de la CCNUCC. Contrairement au mandat de Berlin de $1995^{11}$, qui portait clairement sur l'adoption d'un protocole - le Protocole de Kyoto - le Plan d'action de Bali restait flou et invoquait, de manière quelque peu énigmatique, «un résultat» et l'adoption, pour ce faire, d'une «décision à sa quinzième session ", ${ }^{12}$ ce qui laissait présager plusieurs alternatives : une décision de la Conférence des Parties, prise en vertu des pouvoirs que lui confère l'art. 7.2 de la CCNUCC, pour aborder d'autres actions au titre de la Convention; l'adoption par la Conférence des Parties d'un amendement à la CCNUCC ou à une annexe, pour des actions et/ou engagements supplémentaires des parties de la CCNUCC ; ou l'adoption par la Conférence des Parties d'un nouvel instrument juridique qui complète ou même remplace le Protocole de Kyoto. ${ }^{13}$

Au printemps 2009, il était devenu évident que la Conférence de Copenhague ne pourrait

${ }^{9}$ Id., para 1.

${ }^{10}$ Id., para 1 a et $b$.

${ }^{11}$ Rapport de la Conférence des Parties sur sa première session, tenues à Berlin du 28 mars au 7 avril 1995, FCCC/CP/1995/7/Add.1, Décision 1/CP.1, Mandat de Berlin : Examen des alinéas a) et b) du paragraphe 2 de l'article 4 de la Convention afin de déterminer s'ils sont adéquats, propositions de protocole et décisions touchant le suivi.

${ }_{12}$ Plan d'action de Bali, Supra, note 8, para 1.

${ }^{13}$ Daniel Bodansky, 2009, Legal form of a new climate agreement : Avenues and options, University of Georgia, avril 2009, en ligne (http://www.c2es.org/publications/new-climate-agreement-legal-form), p. 2, consulté le 18 janvier 2016. 
donner tout au plus lieu qu'à un «accord de mise en œuvre» de la CCNUCC, tel que le souhaitait les États-Unis, ${ }^{14}$ et que cet accord ne prendrait pas la forme d'un protocole de la CCNUCC ou ne viendrait pas amender la CCNUCC. Un tel «accord de mise en œuvre» offrirait aux États-Unis la flexibilité nécessaire pour coexister avec un Protocole de Kyoto amendé ou encore pour remplacer complètement le Protocole de Kyoto. L'Accord de Copenhague ${ }^{15}$, qui devait être un «accord de mise en œuvre de la CCNUCC», a été rejeté par la COP. En effet, alors que du 7 au 18 décembre 2009, les yeux du monde étaient rivés sur la capitale danoise, la Conférence, faussement surnommée «Hopenhagen Conference», a donné lieu à la conclusion d'un engagement politique de 3 pages, ficelé à la toute fin de la conférence, et juridiquement non contraignant. Initié par les États-Unis, le Brésil, la Chine, l'Inde et l'Afrique du Sud, il avait été négocié hors de l'AWG-LCA par un groupe de 26 «chefs d'État, chefs de gouvernement, ministres, et autres chefs de délégation présents à la Conférence des Nations Unies sur les changements climatiques de 2009, à Copenhague». II a ensuite été soumis pour adoption à la Conférence des Parties de la CCNUCC. Mais, malgré de longues heures de négociation, la COP a refusé de l'adopter faute de consensus : certains pays, comme la Bolivie, le Venezuela ou le Soudan, s'y sont opposés. La Conférence des parties à la CCNUCC s'est finalement contentée de «prendre note» de cet accord, juste avant que la grand-messe de Copenhague ne prenne fin. ${ }^{16}$

La conférence de Copenhague ne sera toutefois pas un échec total. En effet, après une année délicate, le processus de négociation a repris et le contenu de l'Accord de Copenhague s'est retrouvé, précisé et opérationnalisé, dans une décision de la COP de Cancún, fin $2010{ }^{17}$ Finalement, c'est donc bien l'accord de Copenhague qui structure la période pré 2020, dans le cadre de la Convention, à côté d'un Protocole de Kyoto tout symbolique et non en vigueur. Dans ce cadre extrêmement flexible et respectueux des souverainetés, les Parties, du Nord, comme

\footnotetext{
${ }^{14}$ Bureau of Oceans and International Environmental and Scientific Affairs, U.S. Submission on Copenhagen Agreed Outcome, Washington, DC, May 29, 2009, en ligne: [http://www.state.gov/e/oes/rls/other/2009/124101.htm], page consultée le 18 janvier 2016.
}

${ }^{15}$ CCNUCC, Accord de Copenhague, Décision 1/CP.15, 18 décembre 2009, FCCC/CP/2009/11/Add.1, 30 mars 2010.

${ }^{16}$ Sophie Lavallée, «Le principe des responsabilités communes mais différenciées à Rio, Kyoto et Copenhague. Essai sur la responsabilité de protéger le climat», (2010) 41:1 Études internationales 51-78.

${ }^{17}$ Voir la décision 1/CP.16, Les accords de Cancun: Résultats des travaux du Groupe de travail spécial de l'action concertée à long terme au titre de la Convention, FCCC/CP/2010/7/Add.1, 15 mars 2011. 
du Sud, ont été invitées à faire des promesses de réduction de leurs émissions jusqu'en 2020. Plus de 90 États s'y sont effectivement pliés. Mais les promesses sont largement insuffisantes en nombre et en ambition; elles ne nous placent pas sur la bonne trajectoire. ${ }^{18}$ De surcroît, ni les accords de Cancún ni le Protocole de Kyoto continué, ne vont au-delà de 2020.

\section{L'Accord de Paris : aboutissement et point de départ ?}

À partir de la Conférence de Copenhague, la Chine et les États-Unis, dont le face à face paralysait jusqu'ici les négociations d'un accord post-2012, vont paradoxalement se mettre d'accord pour l'adoption à Paris, lors de la COP 21, d'un accord plus souple que le Protocole de Kyoto découlant certes de la CCNUCC, mais faisant la belle part aux obligations de moyens, tout en déclinant un échéancier de révision périodique des contributions et actions des parties, à l'avenir. La recherche de souplesse s'exprime aussi bien sur la forme (I) que sur le contenu du texte (II).

\subsection{La forme de l'Accord de Paris}

Les États à la Conférence de Paris n'étaient limités dans les actions qu'ils pouvaient négocier que par deux considérations au plan juridique. D'une part, par l'interdiction de négocier un traité qui violerait une norme impérative du droit international, ce qu'on appelle le jus cogens, hypothèse peu probable. ${ }^{19}$ D'autre part, par les règles de procédure et les principes de la CCNUCC elle-même, qui devaient être respectés par la Conférence des Parties. Les règles, destinées à faciliter l'adoption de mesures évolutives dans le temps pour lutter contre le réchauffement climatique, prescrivaient la procédure que la Conférence des Parties devait respecter pour adopter des amendements, des protocoles ou des décisions. ${ }^{20}$ La COP devait aussi respecter les articles 2 et 3 de la Convention, établissant d'une part, l'objectif de celle-ci, et d'autre part, les principes directeurs des responsabilités communes, mais différenciées, de la précaution, et de l'équilibre coût-efficacité des mesures adoptées.

Respectant la procédure requise du consensus et les principes fondateurs de la CCNUCC, la Conférence des Parties a finalement conclu un accord, l'«Accord de Paris», qui répond

\footnotetext{
${ }^{18}$ PNUE, The emissions gap report 2015. What contributions do the INDCs make towards the $2{ }^{\circ} \mathrm{C}$ target? How can the 2030 emissions gap be bridged ?, UNEP, Nairobi, 2015, http://uneplive.unep.org/media/docs/theme/13/EGR 2015 Presentation.pdf.
}

${ }^{19}$ Convention de Vienne sur le droit des traités, art. 53.

${ }^{20}$ CCNUCC, Supra, note 1 , articles 15 à 20 et 22-23. 
formellement à la définition d'un traité, et qui sera à ce titre juridiquement obligatoire pour les États qui décideront de le ratifier. C'est la forme d'engagement la plus solennelle de l'État.

La période de ratification commencera le 22 avril 2016, et l'Accord entrera en vigueur quand 55 États, représentant plus de $55 \%$ des émissions de GES, l'auront ratifié (article 21). II s'intitule «accord», et non pas «convention» ou «protocole» - pour éviter de rappeler le Protocole de Kyoto à ses détracteurs -, mais il s'agit bien d'un traité. Ce traité, selon les juristes américains, n'est pas un treaty au sens du droit constitutionnel américain, mais pourra être considéré plutôt comme un «executive agreement». Ces deux catégories du droit américain correspondent en droit international à des traités. Mais, un «treaty» doit être ratifié par le Sénat, dominé actuellement par les Républicains et très opposés à un tel accord, alors qu'un «executive agreement» peut entrer en vigueur à la suite d'une décision du président. ${ }^{21}$ L'Accord de Paris serait bien un "executive agreement" de la Convention-cadre des Nations unies sur les changements climatiques, laquelle a été ratifiée par les États-Unis le 15 octobre 1992. Ces enjeux du droit constitutionnel américain expliquent la prévalence des obligations de moyens au détriment des obligations de résultat dans l'Accord, ainsi que l'absence de nouvelle obligation financière chiffrée, ou encore de sanctions en cas de non-respect des engagements. La seule sanction sera politique, celle de se voir mettre au banc des «cancres du climat». Mais le retrait du Canada du Protocole de Kyoto a bien démontré que les sanctions prévues par les Accords de Marrakech en 2001, en cas de violation des obligations du Protocole, ne valaient pas leur pesant d'or. ${ }^{22}$

Les revendications des pays développés, de la plupart des pays émergents, et les contraintes du droit interne américain expliquent ainsi que la décision de la $\operatorname{COP}^{23}$ qui adopte formellement l'Accord de Paris, situé en annexe, ne s'en tienne pas là. Cette décision vient également tenter de dynamiser les politiques climatiques sur la période pré-2020, donc dans le cadre des accords de Cancún. Il est en effet essentiel, pour se placer sur la trajectoire définie par l'Accord de Paris (limiter le réchauffement bien en dessous de $2^{\circ} \mathrm{C}$ et même tenter de limiter l'élévation à $1,5^{\circ} \mathrm{C}$ ), de renforcer l'ambition pré-2020. Mais la décision vient aussi compléter et préciser l'Accord de

\footnotetext{
${ }^{21}$ Louis Henkin, «Foreign Affairs and the United States Constitution», Treaties and Other Agreements, Second Edition (1996);L. Henkin, "The President and International Law», AJIL 1986, p. 930-937. D. Bodansky \& Sandra Day O'Connor, Legal options for U.S. acceptance of a new climate change agreement, Center for Climate and Energy Solutions, may 2015, consulté en ligne : [http://www.c2es.org/publications/legal-options-us-acceptance-new-climate-change-agreement], p. 13-14.

22 S. Lavallée et J.-M. Arbour, «Le Canada peut-il invoquer sa propre turpitude pour justifier sa dénonciation du Protocole de Kyoto? ", Le Devoir, 4 janvier 2012.

${ }^{23}$ Décision 1/CP.21, FCCC /CP/2015/L.9, 19 décembre 2015.
} 
Paris sur un grand nombre de points; elle est même quantitativement plus importante que l'Accord (22 pages sur 39 dans la version française). Par exemple, la disposition selon laquelle «avant 2025, la Conférence des Parties agissant comme réunion des Parties à l'Accord de Paris fixe un nouvel objectif chiffré collectif à partir d'un niveau plancher de 100 milliards de dollars par an, en tenant compte des besoins et des priorités des pays en développement» ne figure pas dans le texte de l'Accord, mais dans celui de la décision. De son côté, l'Accord pose les principes fondamentaux qui vont régir les financements climat : qui finance ? Comment ? Quel contrôle ? Ainsi, l'ensemble forme l'accord de Paris, avec une minuscule, un accord qui dépasse largement l'Accord au sens strict. Sur le plan juridique, la décision et l'Accord doivent être lus ensemble et devront également être interprétés l'un à la lumière de l'autre.

Les mêmes contraintes expliquent que les contributions prévues déterminées au niveau national (CPDN), centrales pour atteindre l'objectif de l'Accord de Paris, ne soient pas dans le corps du traité ni en annexe de celui-ci. Elles figureront plutôt dans un registre international dont le secrétariat sera le dépositaire (art. 4 [12]). Elles gagneront ainsi en souplesse et évolutivité. Cela permet de mettre en œuvre aisément la disposition selon laquelle «Une Partie peut à tout moment modifier sa contribution déterminée au niveau national afin d'en relever le niveau d'ambition, conformément aux directives adoptées par la Conférence des Parties agissant comme réunion des Parties à l'Accord de Paris» (art. 4[11]). Mais de ce fait, les contributions n'ont que la valeur d'obligations de moyens. Les Parties doivent prendre toutes les mesures possibles pour s'y conformer, et elles s'engagent, dans l'Accord de Paris, à les établir, les communiquer et les actualiser, et à prendre «des mesures internes pour l'atténuation en vue de réaliser les objectifs desdites contributions» (art. 4[2]).

Ainsi, la forme juridique du résultat de la COP21 a-t-elle été pensée en profondeur pour être subtilement diversifiée, entre ce qui est obligatoire et ce qui ne l'est pas, entre ce qui est à l'intérieur et ce qui reste à l'extérieur (tout en étant étroitement articulé avec ce qui est à l'intérieur). ${ }^{24}$

\footnotetext{
${ }^{24}$ Voir sur les enjeux de la forme juridique, S. Maljean-Dubois, T. Spencer, M. Wemaëre, «La forme juridique du futur accord de Paris sur le climat : enjeux et principales options", Cahiers droits, sciences et technologies, n5/2015, pp. 177-210.
} 


\subsection{Le fond de l'Accord de Paris}

Un traité est toujours juridiquement obligatoire, mais ses dispositions peuvent être plus ou moins contraignantes. Les obligations peuvent être plus ou moins précises, quantifiées, enserrées dans des calendriers ; elles peuvent être auto-exécutoires ou non ; il peut s'agir d'obligations de résultat ou de moyens. L'indicatif («sont», «doivent», «exigent», «ne peuvent pas») peut ou non être préféré au conditionnel («devraient») ou à des formules plus molles («peuvent» ou «s'efforcent»). Les obligations peuvent être atténuées («selon qu'il conviendra», «en fonction des circonstances nationales»). Ainsi, la forme conventionnelle n'est pas une garantie : un traité peut être très mou et peu prescriptif. Plusieurs dispositions de la Convention sur la diversité biologique ${ }^{25}$, par exemple, l'illustrent parfaitement. De la même manière, bien qu'il soit juridiquement un traité, l'Accord de Paris est vague sur bien des points pour lesquels il appelle une concrétisation par des décisions à venir de la COP ou des actions des États. On peut analyser à cette aune l'objectif d'atténuation (2.2.1), les questions de la justice climatique et de la différenciation des «obligations» d'atténuation, de transparence et de financement $(B)$ ou celles relatives à la responsabilité et aux sanctions (2.2.2).

\subsubsection{Un accord ambitieux sur l'atténuation?}

L'article 2 de l'Accord de Paris prévoit que l'élévation de la température moyenne de la planète devra être contenue «nettement en dessous de $2{ }^{\circ} \mathrm{C}$ par rapport aux niveaux préindustriels», et que les efforts devront être poursuivis «pour limiter l'élévation des températures à $1,5{ }^{\circ} \mathrm{C}$ par rapport à l'ère préindustrielle». Cet objectif de 1,5 degré était ardemment souhaité par les pays les plus vulnérables aux changements climatiques, notamment les petits pays insulaires menacés par la montée des mers, qui en faisaient une «ligne rouge» dans leurs négociations. À la COP 21, la «Coalition de la haute ambition» conduite par les Îles Marshall a réussi à rassembler sur cet objectif $1,5^{\circ} \mathrm{C}$, ce qui n'était guère évident avant la conférence. De nombreux pays développés, au titre desquels figurait notablement le Canada, se sont officiellement ralliés à cet objectif plus ambitieux que celui du 2 degrés, ce qui n'a pas manqué de trancher avec la position canadienne de l'ère politique conservatrice. ${ }^{26}$

${ }^{25} 5$ juin 1992, 1760 R.T.N.U. 79.

${ }^{26}$ Sophie Lavallée et Jean-Maurice Arbour, Supra, note 22. 
Le 10 décembre, dans le projet d'accord de Paris, trois alternatives étaient encore débattues par les Parties. L'une des trois options était de limiter la hausse de la température moyenne à $2{ }^{\circ} \mathrm{C}$ par rapport à l'ère préindustrielle, une autre proposait de fixer l'objectif à $1,5^{\circ} \mathrm{C}$ par rapport au seuil préindustriel et la dernière, d'assortir cet objectif de 2 degrés à des efforts en vue d'une possible réduction à $1,5^{\circ} \mathrm{C}$.

On sait que, selon les scénarios établis par le GIEC, pour avoir de bonnes chances de limiter l'augmentation de la température moyenne mondiale à $2{ }^{\circ} \mathrm{C}$, les États doivent réduire leurs émissions mondiales de gaz à effet de serre de 40 à 70 \% par rapport à 2010 d'ici 2050, et les éliminer presque totalement d'ici 2100. On sait aussi que pour limiter la hausse des températures à 1,5 degré, alors que nous affichons déjà une augmentation des températures de l'ordre de 0,86 degré par rapport à l'ère préindustrielle, l'Accord de Paris devrait établir des cibles mondiales chiffrées beaucoup plus ambitieuses de réduction des GES, de l'ordre de 70 à 95 \% en dessous des émissions de 2010, pour l'année 2050. Alors que l'Accord de Paris n'entrera en vigueur qu'en 2020, c'est un objectif titanesque à atteindre, en l'espace de seulement 30 ans. On peut s'interroger sur son réalisme dès lors qu'on ne trouve aucune trace de ces cibles chiffrées et ambitieuses de réduction des GES dans l'Accord de Paris.

Cela était toutefois prévisible et rares sont ceux qui s'en étonnent. On savait déjà, en calculant les contributions nationales divulguées à la suite des conférences de Copenhague (2009) et de Cancún (2010), pour l'horizon 2020, que l'atteinte à long terme d'un objectif de $2^{\circ} \mathrm{C}$ ne serait pas réalisable en s'en tenant là. Ces contributions nationales ne nous mettent pas sur la trajectoire des $2^{\circ} \mathrm{C}$, et encore moins des $1,5^{\circ} \mathrm{C}$. Les États en font d'ailleurs l'aveu, dans la décision accompagnant l'Accord de Paris, lorsqu'ils reconnaissent que le franchissement de la barre des $1,5^{\circ} \mathrm{C}$ est inéluctable, et qu'ils insistent sur "l'urgence de combler l'écart significatif entre l'effet global des engagements d'atténuation pris par les Parties en termes d'émissions annuelles mondiales de gaz à effet de serre jusqu'à 2020 et les profils d'évolution des émissions globales compatibles avec la perspective de contenir l'élévation de la température moyenne de la planète nettement en dessous de $2^{\circ} \mathrm{C}$ par rapport aux niveaux préindustriels et de poursuivre l'action menée pour limiter l'élévation des températures à $1,5^{\circ} \mathrm{C}$ '.

Les États se sont toutefois engagés à réviser et faire progresser de manière évolutive leurs contributions nationales, et ceci dès avant 2020, aidés par un «dialogue de facilitation» qui aura lieu en 2018 (Décision, §20), et auquel le GIEC donnera peut-être un «coup d'accélérateur» en 
produisant juste avant le rapport sur les conséquences régionales d'un dépassement des $1,5^{\circ} \mathrm{C}$ qui lui est demandé (Décision, §21). II faudra toutefois attendre 2023 pour qu'un premier bilan mondial collectif des efforts consentis ait lieu (art. 14 [2]), qui devra inspirer les États dans la définition de leurs contributions nationales quinquennales suivantes (art. 4 [9]). Ce n'est pas le principe de non régression, que des juristes espéraient voir formulé en 2012 dans la Déclaration du Sommet de Rio +20 comme principe du développement durable qui est reconnu textuellement dans l'Accord de Paris, mais bien celui, plus exigeant encore, de «progression», comme pierre angulaire des contributions nationales futures des États (art. 3 et 4[3] et 11)).

En 2015, un rapport sur les trajectoires des émissions de GES du Programme des Nations Unies pour l'environnement affirme qu'environ 450 milliards de tonnes de CO2 (GtCO2) peuvent être émises dans l'atmosphère pour conserver une chance sur deux de demeurer sous les $1,5^{\circ} \mathrm{C}$ de réchauffement global à l'horizon de $2100 .{ }^{27}$ Or, si les États respectent à la lettre leurs engagements de réduction d'émissions, ces 450 milliards de tonnes de CO2 seront émises dès 2024 ; les premières révisions à la hausse de leurs engagements ne devraient intervenir qu'à partir de 2020, soit quatre ans seulement avant que suffisamment de CO2 n'ait été rejeté dans l'atmosphère pour qu'un franchissement de $1,5^{\circ} \mathrm{C}$ soit inévitable.

À Paris, les États ont longuement débattu d'un objectif de long terme en termes de trajectoires d'émission qui vienne préciser comment contenir les températures dans les limites fixées. De ce point de vue, l'article 4 de l'Accord de Paris est bien en retrait de certaines propositions discutées durant la conférence. II exhorte simplement les pays à s'efforcer d'atteindre un plafonnement mondial des émissions de GES «dans les meilleurs délais», émissions qui devront être sur une pente descendante par la suite. Le pic des émissions n'est donc pas daté. Le concept de «neutralité carbone», selon lequel la quantité de GES émise doit être égale à la quantité séquestrée ou compensée, a disparu de l'Accord, comme le réclamait la Chine. II a cédé le pas à la recherche, plus molle, d'un «équilibre entre les émissions anthropiques par les sources, et les absorptions anthropiques par les puits de GES», au cours de la période de 2050 à 2100 .

\footnotetext{
${ }^{27}$ PNUE, The emissions gap report 2015. What contributions do the INDCs make towards the $2^{\circ} \mathrm{C}$ target?, Supra, note 18.
} 


\subsubsection{Un accord de justice climatique?}

Les dispositions d'un traité doivent être lues dans leur ensemble et la différenciation des obligations des pays développés et des pays en développement doit être appréciée à la lumière de chacun des aspects-clés de l'Accord de Paris: les contributions nationales relatives à l'atténuation, leur communication et leur suivi progressif (art. 3, 4, 6) et le financement de l'atténuation et de l'adaptation (art. 9).

L'Accord de Paris reconnaît que le plafonnement des émissions prendra davantage de temps pour les pays en développement parties, ce qui est tout à fait équitable, considérant l'historique mondial des émissions de $\mathrm{CO}_{2}$ et le principe des responsabilités communes, mais différenciées, auquel l'Accord de Paris fait expressément référence dans son préambule (para 3).

Si la justice climatique et le principe des responsabilités communes, mais différenciées, principe fondamental de la CCNUCC (art. 3), ont été au cœur des plus négociations parisiennes, le contenu de l'Accord s'éloigne, de ce point de vue, considérablement de la structure binaire du Protocole de Kyoto, lequel n'exigeait des obligations de réductions des émissions de GES que de la part des pays développés. ${ }^{28}$

Les contributions nationales pour atténuer les changements climatiques, leur communication transparente et leur progression périodique sont des engagements différenciés entre les pays industrialisés, les pays en développement et les pays les moins avancés et pays insulaires en développement (art. 3 et 4 ). Si les pays de la première catégorie "devraient continuer de montrer la voie» en assumant des objectifs de réduction des émissions en chiffres absolus à l'échelle de l'économie, les seconds "sont encouragés à passer progressivement à des objectifs de réduction ou de limitation des émissions à l'échelle de l'économie eu égard aux contextes nationaux différents». (art. 4 [2 à 4]). Les pays les moins avancés et pays insulaires n'ont quant à eux, aucune obligation de communication. L'accord mentionne simplement qu'un soutien

\footnotetext{
${ }^{28}$ Halvorssen, A., "Common, but Differentiated Commitments in the Future Climate Change regime Amending the Kyoto Protocol to include Annex C and the Annex X Mitigation Fund», (2007) 18 Colo. J. Int'l Envtl. L. \& Pol'y, p. 247; Ulrich Beyerlin, «Bridging the North-South Divide in International Environmental Law» (2006) 66 Zeitschrift für ausländisches öffentliches Recht und Völkerrecht 259; B. Müller, Equity in Climate Change: The Great Divide, Oxford Institute for Energy Studies, EV 31, September 2002; Rajamani L., "The Principle of Common but Differentiated Responsibility and the Balance of Commitments under the Climate Regime", (2000) 9 Rev. Eur. Comm. \& Int'l Envt'l L.,p. 120; S. Lavallée, «Le principe des responsabilités communes mais différenciées à Rio, Kyoto et Copenhague. Essai sur la responsabilité de protéger le climat», Études internationales, Vol. XLI, no 1, Mars 2010, 51-78.
} 
financier renforcé leur permettra de prendre des mesures plus ambitieuses dans le futur (art. 4 (5)). Il leur sera difficile de faire des efforts en présentant des contributions nationales s'ils ne sont pas financièrement soutenus de manière prévisible et suffisante. Les pays africains, qui voulaient qu'une mention de l'Afrique et de ses besoins se retrouve partout dans l'accord, n'ont obtenu qu'une mention dans le préambule de la décision. Mais il est à noter que $75 \%$ des pays africains relèvent de la catégorie des «pays les moins avancés» (PMA). Les pays africains ont donc dû se contenter du statut particulier conféré aux PMA dans l'Accord.

Au chapitre du financement, l'approche préconisée par les pays développés, qui était celle d'une «mise à l'échelle» des contributions entre les États, visait évidemment à ne pas limiter le nombre de pays devant fournir de nouvelles ressources financières. Cette question a considérablement ralenti et assombri les négociations. La Norvège, appuyée par l'Union européenne et l'ensemble des pays développés, a fait savoir que, «pour ne pas nuire à l'ambition de mobiliser des ressources au-delà d'un certain montant ${ }^{29}$, elle ne pouvait accepter que l'on renouvelle l'engagement de 100 milliards de dollars américains que les pays développés ont pris à la conférence de Copenhague, ni que l'on chiffre un engagement financier des pays développés dans l'Accord lui-même. Cet argument peut sembler absurde si l'on considère que les pays développés ont réussi, tout au plus, à amasser de 50 à 60 milliards de dollars dans les divers fonds créés avant et à Copenhague, soit bien moins que les 100 milliards de financement par année devant succéder au financement précoce 2010-2012, à atteindre d'ici à 2020, et auxquels se sont engagés les pays développés dans l'Accord de Copenhague, en 2009. ${ }^{30}$

Ainsi, si le chiffre plancher de 100 milliards ne figure pas dans l'Accord, il est toutefois présent dans la décision qui le précède (paragraphes 54 et 115), laquelle établit aussi le principe d'une progression après 2025. Cette décision n'est pas contraignante pour les États qui ratifieront l'Accord. Mais, sans parler de son poids politique, elle aura toutefois une certaine valeur interprétative en ce qu'elle permettra d'«éclairer» les engagements financiers contenus dans l'Accord. II aurait été possible d'adopter dans l'Accord une méthodologie répartissant l'effort de financement entre les pays développés et les pays émergents sur la base d'une différenciation novatrice par rapport à celle adoptée dans les annexes du Protocole de Kyoto. Au lieu de cela, le texte de Paris ne parle que de l'engagement vague des pays développés à soutenir

\footnotetext{
${ }^{29}$ Délégué de la Norvège, Plénière de la Conférence des Parties, 10 décembre 2015.

${ }^{30}$ Accord de Copenhague, Supra, note 15, para. 8.
} 
financièrement les pays en développement (article 9 [1]) en vue de financer prioritairement l'atténuation des effets des changements climatiques et, en cas de ressources accrues, l'atténuation et l'adaptation de manière équilibrée (art. 9 [4]). C'est bien la nécessité de s'accommoder du droit américain en matière de ratification des traités qui a amené les pays à ne pas préciser d'engagement financier dans l'Accord. Ainsi, le Président Barack Obama pourra ratifier l'Accord sans passer par le Sénat, comme nous l'avons vu ci-avant. Quant aux pays émergents - la Chine, l'Inde et la Corée du Sud en tête -, leur voix a été entendue, puisque l'Accord ne fait que les inviter «à fournir ou à continuer de fournir ce type d'appui à titre volontaire» (article 9 [2])

Alors que les pays développés communiquent tous les deux ans des informations à caractère «indicatif» sur les ressources publiques qu'ils ont mobilisées pour soutenir les pays en développement parties, les pays émergents qui fournissent des ressources «sont invité[e]s à communiquer ces informations tous les deux ans à titre volontaire» (art. 9 (5)). Au final, leurs obligations respectives sont peu différenciées, et bien que la communication des transferts soit obligatoire pour les pays développés, un montant «plancher» des contributions globales et respectives des pays développés et émergents est absent de l'accord.

L'assistance financière accordée aux pays en développement n'est pas conditionnée au respect du cadre de transparence des communications de leurs contributions nationales et mesures d'adaptation, mais les articles 13 et 7 de l'Accord n'excluent pas complètement cette conditionnalité. La crainte de voir cette aide financière conditionnée a été exprimée par les pays les moins avancés et les pays insulaires, tout au long de la COP de Paris. Sur la question de la conditionnalité de l'assistance financière, certains pays africains ont partagé les craintes exprimées par l'Arabie Saoudite de voir le respect des droits de l'homme être mentionné dans l'Accord de Paris, craignant que l'aide financière se fasse «à la tête du client». La référence aux droits de l'homme, bien que ne se trouvant pas dans le corps du texte, est présente dans son préambule (para. 11), et pourra servir de source interprétative des dispositions de l'accord.

\subsubsection{Un accord sans sanctions et sans responsabilité ?}

Aucune sanction n'est prévue en cas de non-respect de l'Accord, si ce n'est une sanction politique, celle de se voir mis au banc des «cancres du climat», mais on a vu qu'à ce chapitre, le Canada s'en est relativement bien tiré, allant même jusqu'à se prévaloir de la faculté de se 
retirer du Protocole de Kyoto, ce qui a permis de confirmer que le type de sanctions prévues dans les accords de Bonn-Marrakech ${ }^{31}$, en cas de violations des obligations, qui prévoyaient qu'un État pouvait être pénalisé s'il s'obligeait lors d'une seconde période d'engagement, n'était pas forcément adapté à la vie internationale.

L'Accord de Paris prévoit qu'un mécanisme de promotion et de facilitation du contrôle du respect de ses dispositions sera mis en place (art. 15) qui s'inspirera en partie de l'une des chambres mises en place pour faciliter le respect des dispositions du Protocole de Kyoto, celle de la facilitation. Le texte de l'article 15 de l'Accord de Paris s'éloigne toutefois de celui de l'article 18 du Protocole de Kyoto en ce qu'il spécifie déjà, en amont de la première Réunion des Parties (COP-MOP) qui en précisera les modalités, que ce mécanisme cherchera la facilitation, et fonctionnera «d'une manière qui est transparente, non accusatoire et non punitive». Espérons que cette procédure, dont les détails restent à définir, sera suffisante pour s'assurer du respect des obligations du cadre de transparence mis en place par l'Accord de Paris pour évaluer les progrès périodiques dans la mise en œuvre des contributions nationales, lesquelles - on l'a mentionné - ne se retrouvent pas dans le texte même de l'accord, mais bien dans un registre public distinct, tenu par le secrétariat. À la première réunion des Parties à l'Accord de Paris, la COP examinera des calendriers communs pour les contributions déterminées au niveau national (art. 4 [10]), ce qui en facilitera la communication, l'examen et le suivi, dans le futur. II est aussi envisagé qu'elle adopte des lignes directrices pour encadrer un peu plus les États dans la détermination de leurs contributions.

Enfin, le mécanisme «des pertes et préjudices», réclamé par les pays en développement et obtenu à la Conférence de Varsovie, en 2013, se retrouve dans l'Accord de Paris (art. 8), ce qui est une victoire pour ces pays. II n'est pas confondu avec les mesures concernant l'adaptation, ce qui est souhaitable puisque ce mécanisme sera justement utile lorsque l'atténuation et l'adaptation n'auront pas permis d'éviter la survenance de pertes et dommages. Les négociations au sujet du mécanisme de Varsovie ont toutefois été laborieuses et le texte a été revu jusqu'à la toute fin pour donner toute la flexibilité nécessaire à son développement progressif dans le futur, et pour ne surtout pas en faire un mécanisme de responsabilité ni lui conférer un caractère accusatoire. II s'agira ainsi de développer le «Mécanisme international de Varsovie» dans les années à venir, en tant que mécanisme de "coopération et de facilitation visant à améliorer la compréhension, l'action et l'appui», notamment par la mutualisation des

${ }^{31}$ Décision 24/CP.7, Procédures et mécanismes relatifs au respect des dispositions du Protocole de Kyoto, FCCC/CP/2001/13/Add.3, p. 68. 
risques et l'assurance. Si le mécanisme des pertes et préjudices se retrouve dans l'accord, c'est pour rallier les pays en développement les plus vulnérables qui en avaient fait une condition de leur acceptation de celui-ci. Stratégiquement, ces pays ont soutenu, tout au long des négociations, l'objectif ambitieux de ne pas dépasser un réchauffement de $1,5^{\circ} \mathrm{C}$, position intéressante qui leur permettra peut-être de hâter le moment où ils pourront s'appuyer sur le texte de Paris pour demander aux responsables historiques du réchauffement climatique et aux plus gros émetteurs par habitant de compenser leurs pertes et dommages. Toutefois, le paragraphe 52 de la décision précise que l'article 8 de l'Accord, consacré aux pertes et préjudices, ne «peut donner lieu ni servir de fondement à aucune responsabilité ni indemnisation». Cette disposition a d'ailleurs conduit le Nicaragua à se désolidariser de l'Accord de Paris, dans la déclaration qu'il a faite juste après l'adoption de l'Accord. ${ }^{32}$

\section{Conclusion}

L'évolution des courbes d'émissions depuis la fin du XXe siècle imposait que l'on sorte du cercle restreint des pays de l'annexe I du Protocole de Kyoto. En effet, même si l'ensemble des pays industrialisés - y compris les États-Unis qui ne l'ont pas ratifié - avait respecté leurs engagements de réduction d'émissions de GES dans le cadre du Protocole, l'objectif d'atténuation de celui-ci, soit une réduction globale de l'ordre de 5,2\% en dessous des émissions de 1990, n'aurait pas permis de réaliser l'objectif ultime de la CCNUCC qui est de «stabiliser les concentrations de gaz à effet de serre dans l'atmosphère à un niveau qui empêche toute perturbation anthropique dangereuse du système climatique» (art. 2 CCNUCC). En ce sens, la conclusion à Paris de ce premier accord multilatéral universel engageant tous les pays onusiens à communiquer leurs engagements nationaux tous les cinq ans et à relever progressivement leur niveau d'ambition a été applaudie. À l'aube de la modernité , Thomas Hobbes affirmait que seules des menaces ultimes pouvaient contraindre les individus récalcitrants à s'engager dans le processus d'établissement d'un système politique. ${ }^{33}$ À Paris, le

\footnotetext{
${ }^{32}$ COP 21, débats du 12 décembre au soir, non publiés encore, mais pouvant être suivis en streaming : http://unfccc6.meta-fusion.com/cop21/events/2015-12-12-17-26-conference-of-the-parties-cop-11thmeeting .

${ }^{33}$ Thomas Hobbes, Le Citoyen, Paris, Flammarion, 1982, p. 146.
} 
défi global du réchauffement climatique semble avoir eu un certain effet parmi les États, acteurs certes affaiblis, mais toujours incontournables de la vie internationale. ${ }^{34}$

Déjà lancé à Copenhague, confirmé à Paris, ce processus ascendant et respectueux des souverainetés a donné lieu à la communication, par 188 États à ce jour, de leurs contributions nationalement déterminées, lesquelles couvrent près de $98 \%$ des émissions mondiales. Certes, elles ne nous placent pas sur la trajectoire des $2^{\circ} \mathrm{C}$, et encore moins des $1,5^{\circ} \mathrm{C}$, mais plutôt sur celle des 3 ou $3,5^{\circ} \mathrm{C}$. Mais cela représente une avancée significative; les scénarios au fil de l'eau laissaient plutôt envisager 4,5 ou $6^{\circ} \mathrm{C}$, ce qui dessinerait un monde bien différent. Maintenant que tout le monde ou presque est «à bord», il reste à relever le niveau d'ambition des contributions nationales, fortement et au plus vite.

Certains outils sont là dans l'accord de Paris. Mais les COP à venir vont-elles les concrétiser ? Les États vont-ils les utiliser ? Les financements promis vont-ils être alloués et accompagner la transition des pays en développement? II faut en effet espérer que les transferts financiers suivront et qu'ils feront mentir des pays qui, comme la Barbade, exprimaient ainsi leurs craintes à Paris : «Les changements climatiques menacent notre stabilité économique et notre survie en tant qu'État insulaire. Nous n'apposerons pas notre signature à un accord qui marquerait l'extinction de nos peuples. » Une autre question importante est celle de savoir si ce défi colossal pourra être relevé sans prix mondial et taxation du carbone ${ }^{35}$ Seul l'avenir le dira. II est néanmoins regrettable que les Parties ne se soient pas entendues pour fixer ce prix. Toutefois, les divergences de vues sur cette question auraient été susceptibles d'entraîner un blocage des négociations. Concentré sur les objectifs, l'Accord de Paris ne fait en réalité guère avancer sur le «comment»: comment opérer collectivement la transition énergétique vers un monde décarboné ? De ce point de vue, il ne résout pas le "schisme de réalité» mis en évidence par Amy Dahan et Stefan Aykut, résultant d'un «décalage croissant entre, d'un côté, une réalité du monde, celle de la globalisation des marchés, de l'exploitation effrénée des ressources d'énergie fossiles et des États pris dans une concurrence économique féroce et s'accrochant plus que jamais à leur souveraineté nationale et de l'autre, une sphère des négociations et de la

\footnotetext{
${ }^{34}$ F. Cerutti, «Le réchauffement de la planète et les générations futures», Pouvoirs, n¹27 - Droit et environnement - novembre 2008 - p.107-122, p. 108-109.

${ }^{35}$ Olivier Milman, «James Hansen, father of climate change awareness, calls Paris talks 'a fraud'», The Guardian, 12 décembre 2015.
} 
gouvernance qui véhicule l'imaginaire d'un 'grand régulateur central' apte à définir et à distribuer des droits d'émission, mais de moins en moins en prise avec cette réalité extérieure». ${ }^{36}$

L'optimiste considèrera que l'Accord de Paris pourrait lancer une nouvelle dynamique, puisqu'il met fin à plus d'une décennie de blocage des négociations, blocage qui avait conduit à évoquer une crise du multilatéralisme climatique. Le déroulement de la Conférence de Paris a indiscutablement fait progresser la confiance dans ce processus, et la confiance mutuelle entre les Parties. Déjà timide à l'origine, cette confiance avait été ruinée lors de la Conférence de Copenhague. Ces progrès laissent espérer un déroulement moins chaotique des négociations maintenant lancées pour l'opérationnalisation de l'Accord de Paris. Mais le processus reste fragile. En outre, il ne faut pas oublier que l'ambition des politiques climatiques dépend aussi d'autres processus et institutions, comme s'agissant des combustibles de soute, puisque les États ne se sont pas entendus à Paris pour taxer les émissions des transports aériens et maritimes internationaux ${ }^{37}$. Le schisme de réalité est là aussi bien présent.

À ces différents égards, et en tant que nouvelle construction dans l'édifice complexe qu'est le régime climatique de la CCNUCC, l'Accord de Paris semble davantage marquer le commencement d'une nouvelle ère de diplomatie climatique qu'un aboutissement. Affaire à suivre, donc !

\footnotetext{
${ }^{36}$ A. Dahan Dalmedico, S. Aykut, Gouverner le climat, 20 ans de négociations internationales, Presses de Sc. Po, Paris, 2015, pp. 399-400.

${ }^{37} \mathrm{~V}$. Trien Lam, "Les enjeux juridiques de l'intégration des pays en développement dans le régime climatique post-Kyoto», (2010) 23 RQDI 31, p. 88-89; S. Lavallée, "Responsabilités communes mais différenciées et protection de l'environnement: une assistance financière en quête de solidarité?", L'équité en droit international de l'environnement, Les Cahiers de droit, Vol. 55, no 1, mars 2014, 139, pp. 186-187.
} 